\title{
Hepatitis C Virus Recombinant Immunoblot Assay
}

National Cancer Institute

\section{Source}

National Cancer Institute. Hepatitis C Virus Recombinant Immunoblot Assay. NCI

Thesaurus. Code C95525.

An assay used to verify the presence of anti-HCV antibodies in a blood sample. Serum is incubated with a membrane strip on which four recombinant viral proteins are attached. The immunoblot is considered positive if two or more proteins react and indeterminate if only one positive band is detected. 